\title{
On the elasticity of Copper(II)acetylacetonate crystals
}

ARISING FROM A. Worthy et al. Nature Chemistry https://doi.org/10.1038/nchem.2848 (2018).

Susobhan Das ${ }^{\mathrm{a}}$, Gerrit Vreeman ${ }^{\mathrm{b}}$, Upadrasta Ramamurty ${ }^{\mathrm{c}, \mathrm{d} *}$, Changquan Calvin Sun ${ }^{\mathrm{b} *}$, C. Malla Reddy*

${ }^{a}$ Department of Chemical Sciences, Indian Institute of Science Education and Research (IISER) Kolkata, Mohanpur 741246, India

${ }^{b}$ Pharmaceutical Materials Science and Engineering Laboratory, Department of Pharmaceutics, College of Pharmacy, University of Minnesota, Minneapolis, Minnesota 55455, United States

'School of Mechanical and Aerospace Engineering, Nanyang Technological University, Singapore 639798, Republic of Singapore

dinstitute of Materials Research Engineering, Agency for Science, Technology and Research, Singapore 138634, Republic of Singapore

Email:uram@ntu.edu.sg;sunx0053@umn.edu; cmallareddy@gmail.com

Structure-property correlations in a wide variety of elastic molecular crystals indicate that (a) corrugated structures that can interlock readily (prevent plastic deformation either through geometrical or energetic considerations) and (b) a multitude of weak and dispersive intermolecular interactions that would act as structural buffers through easy rupture and reformation during deformation, are structural features that impart a crystal the ability to accommodate large elastic strains. ${ }^{1-5}$ Based on their studies on copper(II)acetylacetonate crystals (1, hereafter), Worthy et al. claimed that these criteria are "incorrect". ${ }^{6}$ In order to examine their claim critically, we conducted detailed experiments on crystals of 1 . Our flexure experiments show that they indeed readily undergo plastic deformation when bent on the (101) face, which contradicts Worthy et al. 's assertion that the (101) face is highly elastic. Therefore, the crystal 1 is not an ideal model system for rejecting the prevailing - and widely acceptedmolecular mechanisms for exceptional elastic flexibility of organic crystals.

Upon stressing, all solids deform elastically first, before the onset of either plastic deformation or brittle failure. Depending on the extent to which a molecular crystal exhibits these characteristics, it is broadly termed elastic, plastic, or brittle. ${ }^{1}$ In particular, elastic crystals are those that can accommodate extraordinarily large elastic strains. 

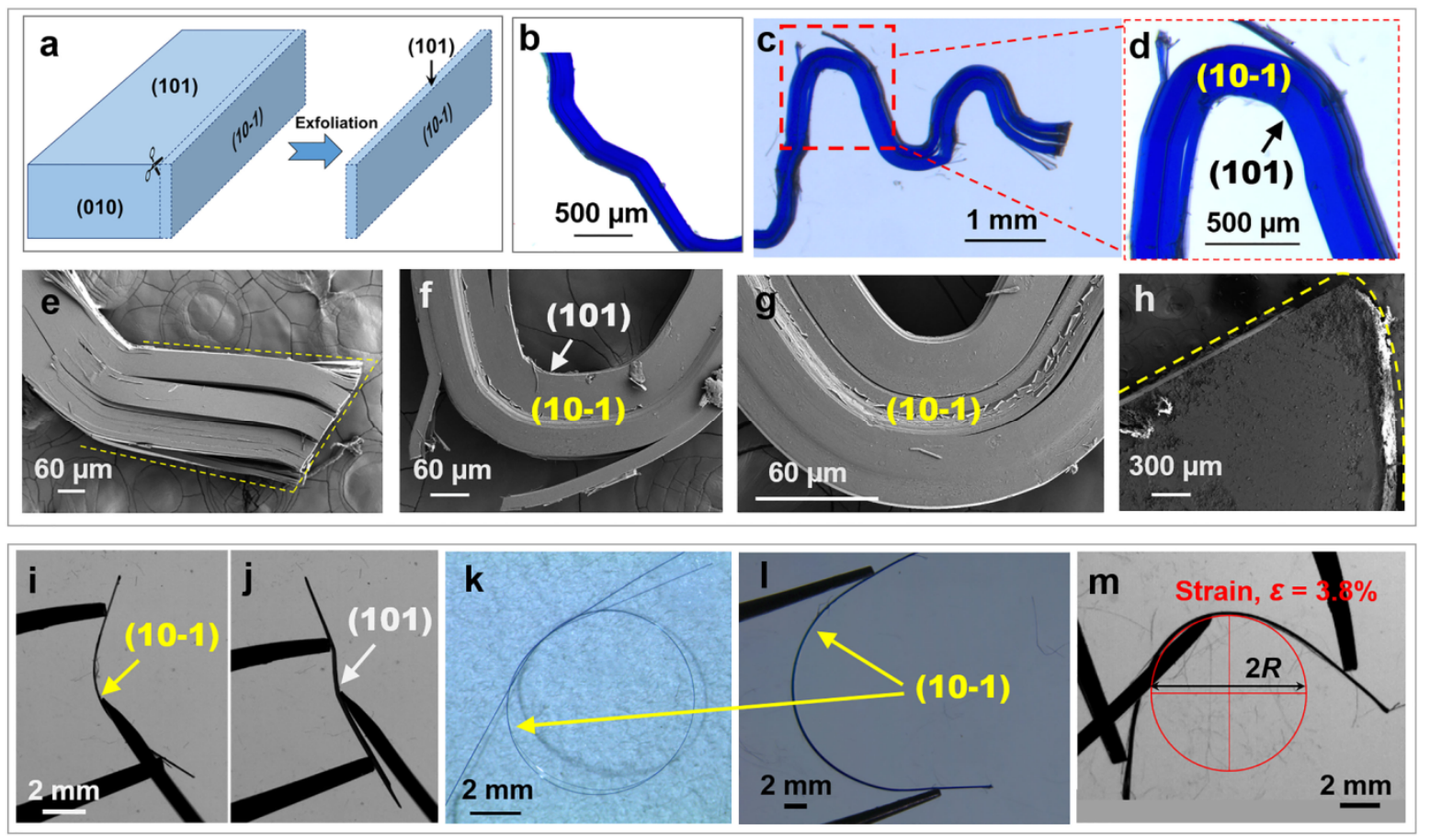

Figure 1. Morphology and qualitative mechanical behaviour of crystal 1. (a) Schematic representation of as-grown crystals' morphology and exfoliation of slices. (b), (c) and (d) Plastic deformation on (101) in thick crystals. Notice the delamination at the strained regions. (e-g) Crystals showing plastic bending and (h) a pellet made using bulk microcrystalline

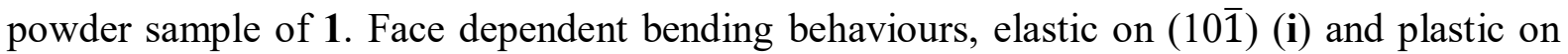

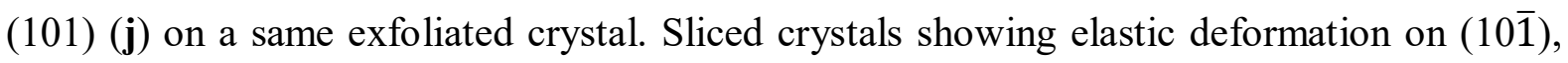
resulting a crystal knot (k), half circle (I) and another half circle with indicated elastic strain limit (m). Note that panels (b-d, i-m) are optical images and (e-h) are scanning electron micrographs (SEMs).

We obtained 1-3 cm long needle type crystals of 1, grown along $b$-axis (Fig. 1a), with morphologies consistent with those of Worthy et al. Our qualitative mechanical tests, with forceps and a metal pin, revealed that the as-grown crystals of 1 undergo plastic deformation readily when bent on the major (101) face with the flexural strain exceeds $\sim 1 \%$ (see Figs. 1b$\mathrm{g}$ and Figs. S1, S2 in SI). Plasticity is accompanied by delamination parallel to (101) (Figs.1bg and Figs. S1c). The irreversible morphological changes are similar to those observed in other well-known plastically bent crystals, such as $\mathrm{C}_{6} \mathrm{Cl}_{6} \cdot{ }^{7-9}$ These crystals of 1 can be easily

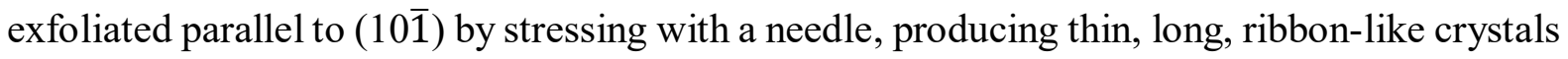

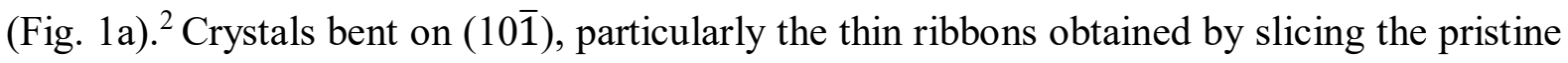
crystals, showed remarkable elastic flexibility (Figs.1k-m and Figs. S1e-g,i), unlike the (101)

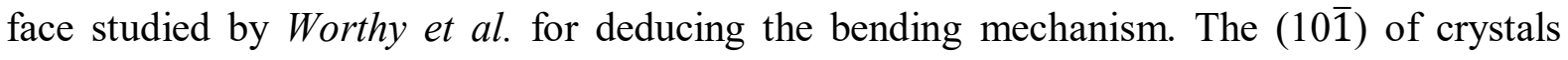
accommodated significant strains (larger than $4 \%$ ) (Fig. S3) and could be tied into knots having a small radius, confirming its superior elastic nature as compared to (101) (Figs. 1k,

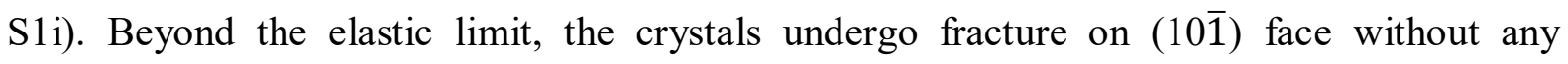
appreciable plastic deformation (Fig. S1). 
Worthy et al. overlooked these distinct mechanical responses of the two faces. They stated that the (101) face is more elastic than (10-1) face and that these observations are in agreement with the lower elastic moduli of $(101)$ face $(4.8-6.9 \mathrm{GPa})$ than $(10-1)$ face $(11.3-13.8 \mathrm{GPa})$. Indeed, the relatively lower elastic modulus of (101) confirms its compliant nature, but not necessarily higher elasticity, contrary to what is stated by Worthy et al. Careful three point bending tests on crystals with different orientations would have allowed the quantitative assessment of the two faces. But, Worthy et al. failed to mark the faces of bent crystals used for several experiments, leading to ambiguity in their analysis. For instance, their crystal-knot

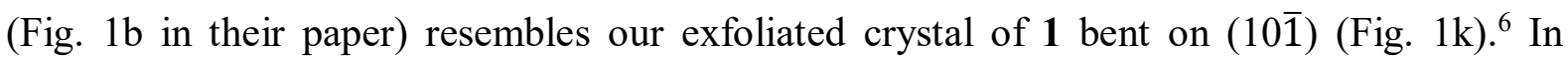
contrast, when bending the as grown crystals on their (101) face, we could not form an elastic knot as they readily underwent plastic deformation. Since the micro focused X-ray diffraction studies by Worthy et al. were performed on crystals of $\mathbf{1}$ bent on the predominantly plastic

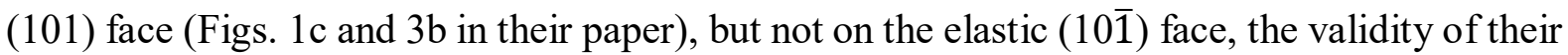
proposed mechanism and conclusions are in question. ${ }^{6}$

Additionally, we ascertained the plastic nature of crystals 1 by measuring the stress transmission ratio (STR, axial compression stress to radial die wall stress) of its bulk powder during die compression. STR is a widely used measure in the pharmaceutical industry for assessing the tabletability of the active pharmaceutical ingredients. A more plastic material can transmit stress from the axial direction to the radial direction effectively, exhibiting a higher STR. ${ }^{10}$ The observed stress transmission behaviour of $\mathbf{1}$ resembles that of the well-known highly plastic microcrystalline cellulose, ${ }^{11}$ confirming the predominantly plastic nature of crystals of 1 during die compression (see SI, Fig. S4).

Worthy et al. performed their structural investigations only within a small elastic strain. On that basis, they proposed a molecular rotation mechanism, which was used to rationalize elongation (outer arc) and compression (inner arc) of the bent crystals. However, their assertion that the observed structural changes in $\mathbf{1}$ can be applied to explain exceptionally high elastic strain in some molecular crystals is incorrect. Especially, the molecular rotation features in 1, cannot form a basis for rejecting the other structural requirements postulated for highly elastic molecular crystals. ${ }^{1-5,12-14}$ We further contend that the rotation model is applicable only to crystals formed by non-spherical species. For instance, rotations by nearly spherical molecules do not result in any expansion or compression of crystals. Hence, any elasticity observed in such systems cannot be explained by the "rotation" based mechanisms. Instead, small linear displacements of constituents are sufficient to rationalize their elastic compression and expansion, for instance, as seen in single crystals of elemental metals. ${ }^{15}$

Thus, interlocking between adjacent molecular layers, either mechanically or energetically, remains a necessary condition for a molecular crystal to exhibit a high elastic limit. It follows that increasing the strength of interactions that operate at longer distances between adjacent molecular planes facilitates larger elastic strain limits in crystals. Such molecular crystals exhibit both higher rigidity, by more effectively hindering plastic deformation, and greater elastic flexibility, by accommodating more changes in molecule positions that can recover after withdrawal of the external stress. ${ }^{16}$ 
In summary, although the study by Worthy et al. sheds light on the molecular level response of crystals of 1 to external stresses within a small elastic strain, it fails to emphatically provide a valid basis for casting aspersions on the validity of the earlier postulated structural features for molecular crystals to exhibit high elastic flexibility. This clarification is important for the following two important reasons: (i) design of exceptionally elastic molecular materials for potential technological applications, and (ii) set the record straight for this exciting area of scientific research.

\section{References}

1. Saha, S., Mishra, M. K., Reddy, C. M. \& Desiraju, G. R. From molecules to interactions to crystal engineering: mechanical properties of organic solids. Acc. Chem. Res. 51, 2957-2967 (2018).

2. Hayashi, S. \& Koizumi, T. Mechanically induced shaping of organic single crystals: facile fabrication of fluorescent and elastic crystal fibers. Chem. Eur. J. 24, 8507-8512 (2018).

3. Ghosh, S., Mishra, M. K., Kadambi, S. B., Ramamurty, U. \& Desiraju, G. R. Designing elastic organic crystals: highly flexible polyhalogenated N-benzylideneanilines. Angew. Chem. Int. Ed. 54, 2674-2678 (2015).

4. Ahmed, E., Karothu, D. P. \& Naumov, P. Crystal adaptronics: mechanically reconfigurable elastic and superelastic molecular crystals. Angew. Chem. Int. Ed. 57, 8837-8846 (2018).

5. Ghosh, S. \& Reddy, C. M. Elastic and bendable caffeine cocrystals: implications for the design of flexible organic materials. Angew. Chem. Int. Ed. 51, 10319-10323 (2012).

6. Worthy, A. et al. Atomic resolution of structural changes in elastic crystals of copper(II) acetylacetonate. Nat. Chem. 10, 65-69 (2018).

7. Reddy, C. M. et al. Structural basis for bending of organic crystals. Chem. Commun. 3945-3947 (2005).

8. Panda, M. K. et al. Spatially resolved analysis of short-range structure perturbations in a plastically bent molecular crystal. Nat. Chem. 7, 65-72 (2015).

9. Reddy, C. M., Padmanabhan, K. A. \& Desiraju, G. R. Structure-property correlations in bending and brittle organic crystals. Cryst. Growth Des. 6, 2720-2731 (2006).

10. Ridgway, K., Glasby, J. \& Rosser, P. H. The effect of crystal hardness on radial pressure at the wall of a tabletting die. J. Pharm. Pharmacol. 21, 24S-29S (1969).

11. Hu, S., Mishra, M. K. \& Sun, C. C. Twistable pharmaceutical crystal exhibiting exceptional plasticity and tabletability, Chem. Mater. 31, 3818-3822 (2019).

12. Kusumoto, S. et al. Elastic crystalline fibers composed of a nickel(II) complex. Inorg. Chem. 60, 1294-1298 (2021).

13. Mishra, M. K. \& Sun, C. C. Conformation directed interaction anisotropy leading to distinct bending behaviors of two ROY polymorphs. Cryst. Growth Des. 20, 4764-4769 (2020). 
14. Funamori, Y. et al. Large elastic deformation of $\mathrm{C}_{60}$ nanowhiskers. Carbon 169, 65-72 (2020).

15. Nye, J. F. Physical Properties of Crystals: Their Representation by Tensors and Matrices (Clarendon Press, 1985).

16. Mukherjee, A. \& Desiraju, G. R. Halogen bonds in some dihalogenated phenols: applications to crystal engineering. IUCrJ 1, 49-60 (2014).

\section{Acknowledgements}

CMR thanks DST, New Delhi for a Swarnajayanti Fellowship (DST/SJF/CSA-02/2014-15) and SERB for funding (No: EMR/2017/005008). Fellowship for SD (CSIR-SRF; File No09/921(0162)-2017-EMR-I) is acknowledged and funding from the National Science Foundation through grant number IIP- 1919037 is gratefully acknowledged for partially supporting GV.

\section{Author contributions}

Material synthesis, crystallization and all mechanical manipulation of crystals was done by SD. Face dependent behaviour and face indexing on pristine crystals was further independently checked by GV. Die compression studies on powders were done by GV under the supervision of CCS. CMR, CCS and UR analysed all the results and co-wrote the manuscript taking inputs from all the authors.

\section{Competing interests}

The authors declare no competing interests.

\section{Additional information}

All additional data is available in the supplementary materials. 


\section{Supplementary Information}

\section{On the elasticity of Copper(II)acetylacetonate crystals}

ARISING FROM A. Worthy et al. Nature Chemistry https://doi.org/10.1038/nchem.2848 (2018).

Susobhan Das ${ }^{\mathrm{a}}$, Gerrit Vreeman ${ }^{\mathrm{b}}$, Upadrasta Ramamurty ${ }^{\mathrm{c}, \mathrm{d} *}$, Changquan Calvin Sun ${ }^{\mathrm{b} *}$, C. Malla Reddy ${ }^{\mathrm{a} *}$

Supplementary contents:

Supplementary note $1-2$

Supplementary figures S1-S4

References 


\section{Supplementary note 1: Crystal growth, mechanical response of as-grown and exfoliated crystals.}

Crystal Growth. The copper(II)acetylacetonate complex was prepared by following the known method. ${ }^{17}$ Blue long needle shaped crystals were obtained from warm chloroform solution by using slow evaporation crystallization technique.

Mechanical response of as-grown and exfoliated crystals. Blue long needle-like as grown single crystals of $\mathrm{Cu}(\mathrm{acac})_{2}, \mathbf{1}$, mainly consist of two faces, wide (101) and thin (10 $\left.\overline{1}\right)$ faces [Fig. S1a-(i)]. As-grown crystals tend to fracture when stressed locally on (10 $\overline{1})$ face [Figs. S1a-(ii-iii)], but undergo facile plastic deformation when stressed on (101) face [Figs. S1a(iv)\&(v), S1b]. The pristine crystals, upon pocking on (101) face by a needle, exfoliate parallel to $(10 \overline{1})$ planes, resulting in thin, long ribbon-like slices (Fig. 1a, main draft). Such exfoliation reduces the area of (101), hence the $(10 \overline{1})$ appears larger (Fig. 1a, main draft). The exfoliated crystals of 1 show plastic deformation on (101) (Figs. S1c,d), similar to the pristine crystals,

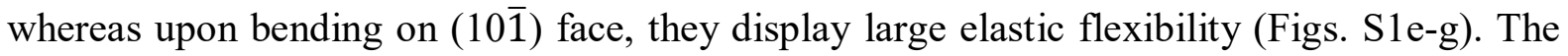
crystals also can be twisted plastically around the growth axis (Fig. S1h). Knots could be readily formed from thin, long ribbon type crystals obtained from exfoliating pristine crystals (Fig. S1i).

Supplementary note 2: Plasticity assessment. The bulk powders of 1 transmits axial stress to radial die wall more effectively than the highly plastic microcrystalline cellulose (MCC $)^{11}$ (Fig. S4a). For a liquid, the stress transmission ratio (STR, i.e., the slope of the linear portion of the curve) is unity. A solid with a STR value closer to unity is more plastic. By the measure of STR, the plasticity of $\mathbf{1}$ is comparable to that of the highly plastic MCC. Additionally, $\mathbf{1}$ exhibits a high propensity of punch sticking during compression, which is also indicative of high plasticity. ${ }^{18}$ 

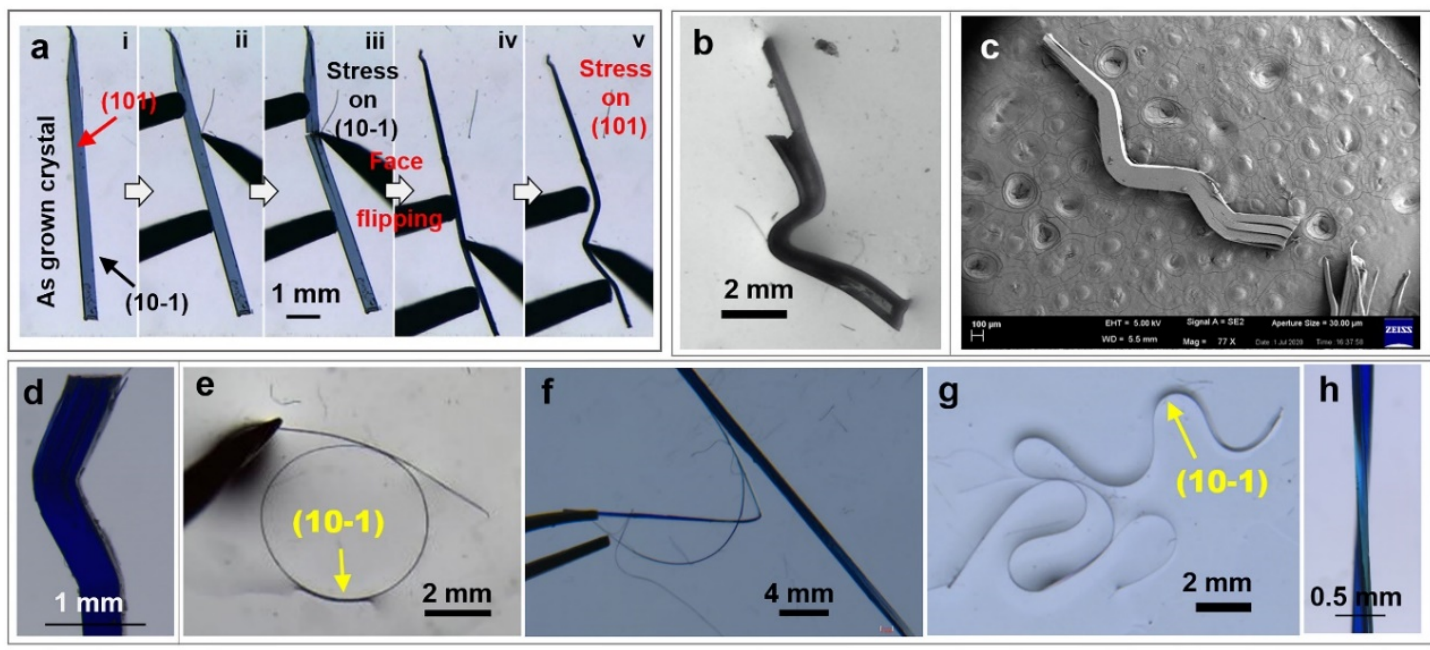

i)

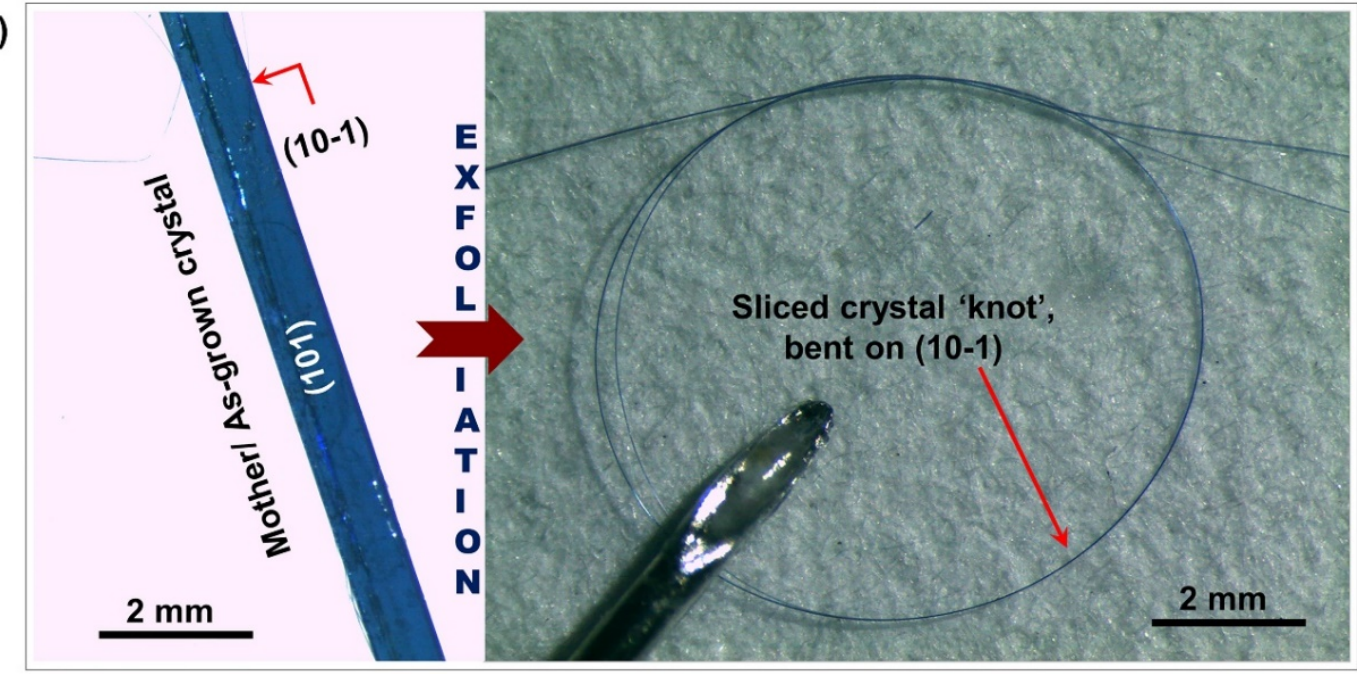

Figure S1. Face-dependent mechanical responses of as-grown and exfoliated crystals of 1. (a)

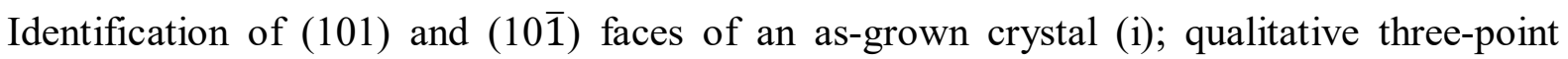

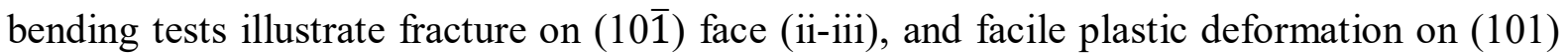
faces (iv-v). (b) A thick version of an as-grown crystal with facile plastic bending. (c,d) Irreversible bending deformation with change in interfacial angle at the top edge of an exfoliated crystal, comparable to many well-known plastically deformable crystals. (e) Elastic loop formed using an exfoliated crystal. (f) Elastic deformation of an exfoliated ribbon separated from an as-grown crystal. (g) Exceptional elastic bending in a very thin exfoliated crystal. (h) Crystal with an irreversible twisting around the needle axis. (i) Comparison of a

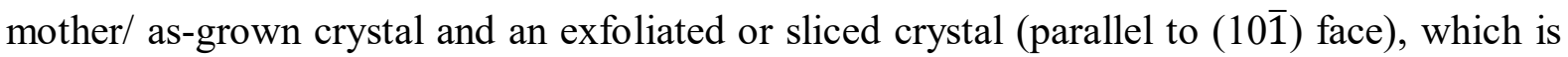
capable of being deformed into a Crystal Knot. 
a)

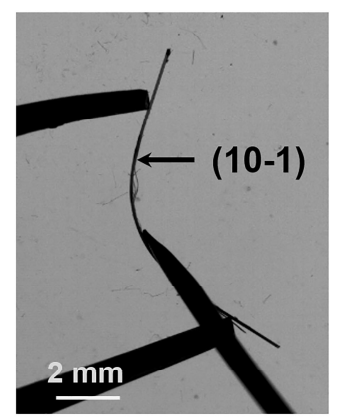

c)

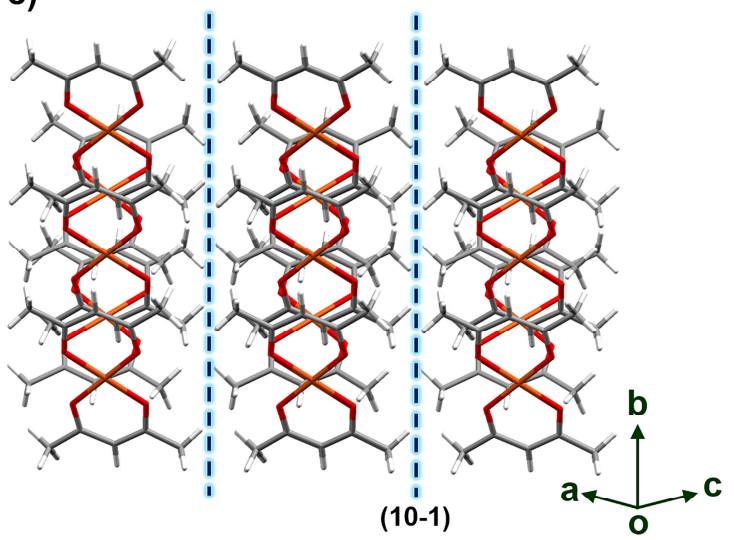

b)

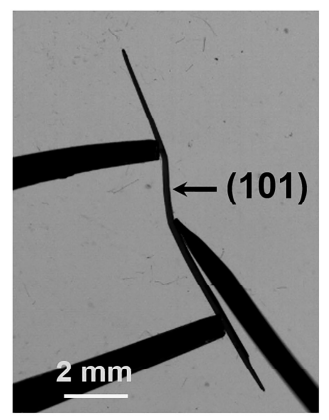

d)

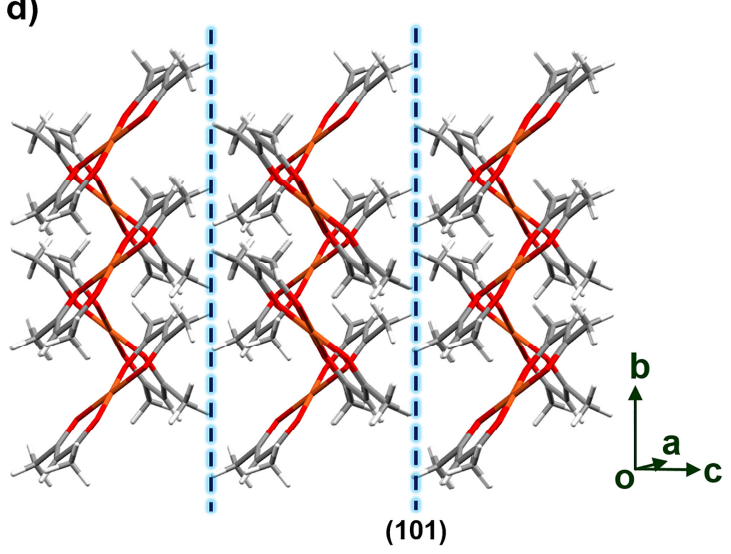

e)

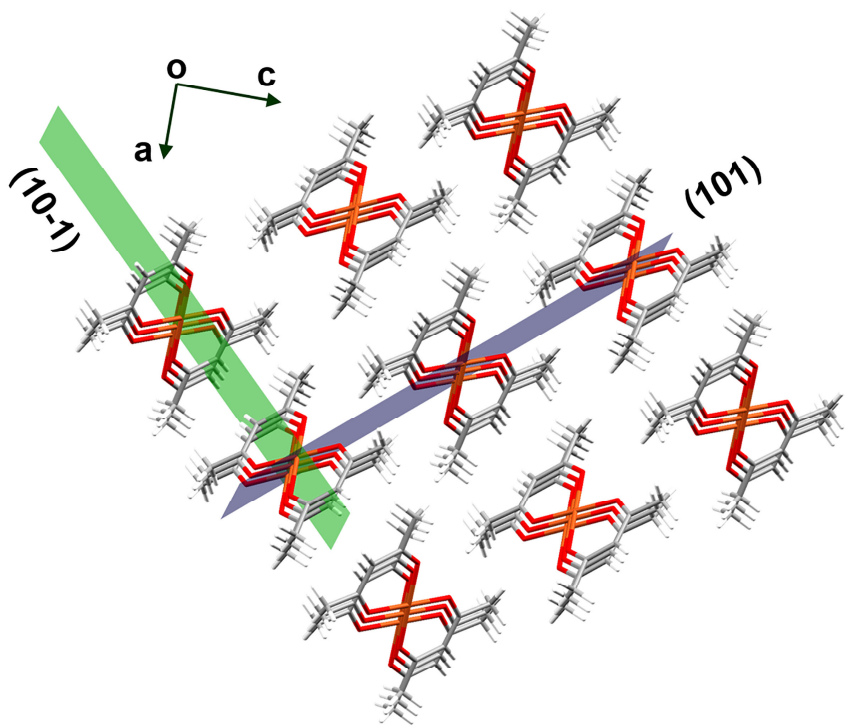

Figure S2. Different mechanical behaviours observed from different faces, (a) (10 $\overline{1})$ and (b) (101) and the crystallographic packing diagrams marked with corresponding crystallographic planes, (c) and (d), respectively. (e) A crystal packing viewed down the $b$-axis with

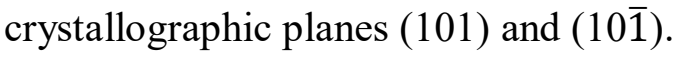


a)

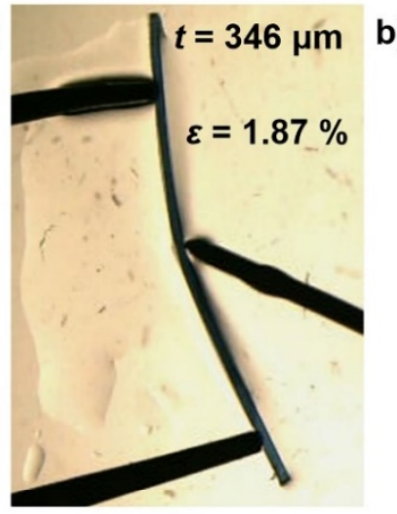

b)

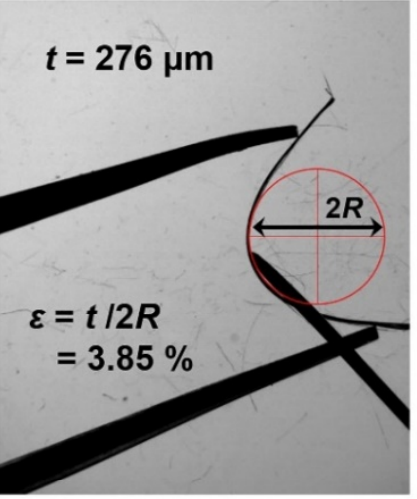

c)

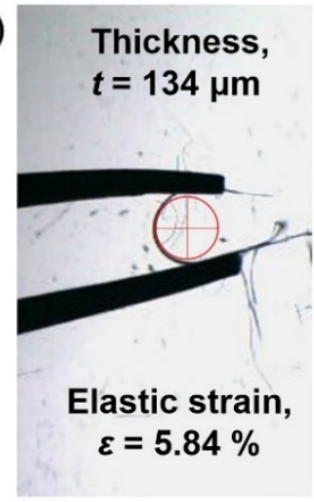

d)

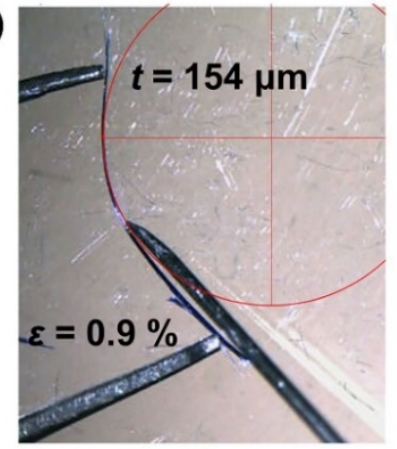

e)

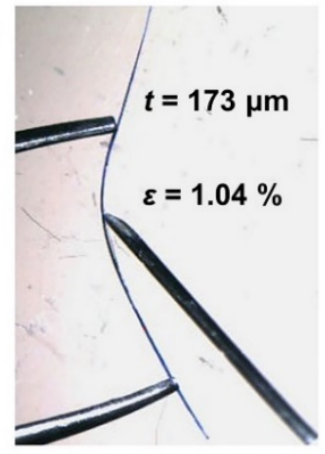

f)

\section{Bending Strain Calculation:}

Crystal thickness, $t=\mathrm{x} \mu \mathrm{m}$

Diameter of curvature, $2 R=y \boldsymbol{m}$

So, \% of elastic strain,

$\varepsilon=(t / 2 R) * 100=(\mathrm{x} / \mathrm{y}) * 100=\mathrm{z} \%$

Figure S3. Calculation of strain in exfoliated crystals of 1 on different faces. (a-c) Elastic bending strain on $(10 \overline{1})$ of the crystals with varied thickness before fracture. (d,e) Elastic bending strain on (101) of crystals before plastic deformation sets in. (f) Calculation method used following the Euler-Bernoulli beam-bending theory of strain on perturbed crystals.

a

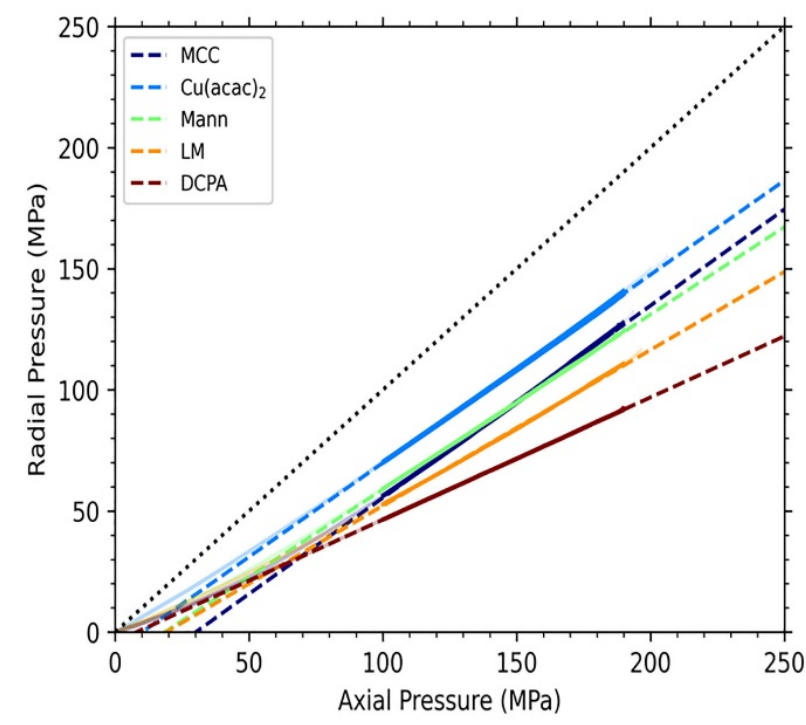

b

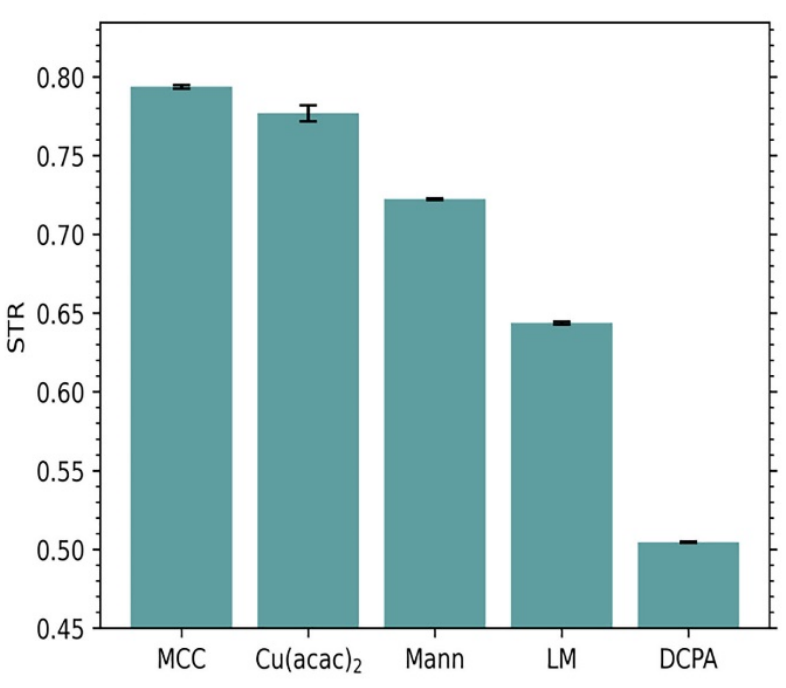

Figure S4. Plasticity of 1 quantified by radial die wall stress transmission in comparison to common materials, including lactose monohydrate (LM), microcrystalline cellulose (MCC), and dibasic calcium phosphate (DCPA). (a) Radial pressure as a function of axial pressure during the course of compression. The dashed line represents $100 \%$ transmission of axial stress to the die wall. (b) Stress transmission ratios $(\mathrm{n}=3)$. 


\section{References.}

17. Glidewell, C. Metal Acetylacetonate Complexes: Preparation and Characterisation. In Inorganic Experiments, Third Edition; Woolins, D., Ed.; Wiley-VCH: Weinheim, Germany, 2010; pp 109-119.

18. Paul, S. et al. Dependence of punch sticking on compaction pressure - roles of particle deformability and tablet tensile strength, J. Pharm. Sci., 106, 2060-2067 (2017). 